\title{
Adaptação do Questionário dos Fundamentos Morais para o Português
}

\author{
Alexandre Magno Dias Silvino - Universidade de Brasília, Brasília, Brasil e Instituto Nacional de Estudos e \\ Pesquisas Anisio Teixeira, Brasilia, Brasil \\ Ronaldo Pilati - Universidade de Brasilia, Brasília, Brasil \\ Victor Nahuel Keller - Universidade de Brasília, Brasilia, Brasil \\ Elena Pinheiro Silva - Universidade de Brasilia, Brasília, Brasil \\ Aline Fernandes de Paula Freitas - Universidade de Brasilia, Brasilia, Brasil \\ Juliana Nunes Silva - Universidade de Brasilia, Brasilia, Brasil \\ Maíra Fernandes Lima - Universidade de Brasilia, Brasilia, Brasil
}

\begin{abstract}
Resumo
O estudo objetivou avaliar as evidências de validade de uma versão adaptada para o português do Questionário de Fundamentos Morais que analisa a relevância de cinco fundamentos morais para o julgamento moral: dano/cuidado, justiça/reciprocidade, pertencimento/lealdade, autoridade/respeito e pureza/santidade. Participaram 532 pessoas, considerando-se todas as fases. Os procedimentos incluíram tradução/retradução, análise da adequação nos idiomas inglês e português e análises fatoriais exploratórias. As análises indicaram que uma estrutura de dois fatores foi a melhor solução encontrada. O primeiro fator Individualizante $(\alpha=0,91)$ e o segundo Coesivo $(\alpha=0,87)$, solução compatível com a teoria ao agrupar os fundamentos de dano e justiça em contraposição aos demais. Foram encontradas evidências de validade convergente entre os fundamentos de coesão e uma medida de religiosidade. Conclui-se que o instrumento distingue um padrão de escolha moral de individualização ou ligação, o fundamento mais influente e sua estrutura fatorial corrobora àquela dos autores da versão original.

Palavra-chave: moral, teoria dos fundamentos morais, avaliação psicológica
\end{abstract}

\section{Adaptation of the Moral Foundations Questionnaire to Brazilian Portuguese}

\begin{abstract}
The study aimed to evaluate evidences of the validity of the Moral Foundations Questionnaire in a version adapted to Portuguese, considering five foundations for moral judgment: harm/care, fairness/reciprocity, ingroup/loyalty, authority/respect and purity/sanctity. 532 people were involved in this study throughout all of its phases. The procedures included translation/ retranslation, analysis of adequacy on both languages and exploratory factorial analysis. The analysis indicated that the best solution found is a structure composed of two factors. The first one being the individualizing factor $(\alpha=0.91)$ and, the second, the cohesive factor $(\alpha=0.87)$, a solution which is compatible with the theory when the foundations of damage and justice are grouped together as opposed to the others. Evidences of convergent validity were found between the cohesion foundation and a religiosity measure. Concluding, the instrument distinguishes a pattern of moral choice of individualization, the most influential foundation, and its factorial structure corroborates the original version.

Keywords: morality, moral foundations theory, psychological assessment
\end{abstract}

\section{Adaptación del Cuestionario de Fundamentos Morales para el Portugués}

\begin{abstract}
Resumen
El estudio tuvo como objetivo evaluar las evidencias de validez de una versión adaptada para el portugués del Cuestionario de Fundamentos Morales, que analiza la pertinencia de cinco fundamentos para el juicio moral: daño/cuidado, justicia/reciprocidad, pertenencia/lealtad, autoridad/respeto y pureza/santidad. Participaron 532 personas considerándose todas las fases. Los procedimentos incluyeron: traducción y retraducción, análisis de adecuación en los idiomas inglés y portugués y análisis factorial exploratorio. Los análisis indicaron una estructura de dos factores como la mejor solución encontrada: a) individualización ( $\alpha=$ $0,91)$ y cohesión $(\alpha=0,87)$, solución compatible con la teoría al agrupar los fundamentos de daño y justicia en contraposición a los demás. También se encontraron evidencias de validez convergente entre los fundamentos cohesión y una medida de religiosidad. Para finalizar se distingue un padrón de elección moral de indiviluazación o vínculo, el fundamento más influyente, y su estrucuta factorial corrobora a de los autores de la versión original.

Palabras-clave: moral, teoría de fundamentos morales, evaluación psicologica
\end{abstract}

Temas relacionados a dilemas morais têm sido cada vez mais comuns no cotidiano da era da informação. Assuntos outrora evitados ganham mais espaço com a evolução constante das novas tecnologias que oportunizam canais de debates francos e abertos como casamento gay, aborto, pesquisa de células-tronco, eutanásia, etc. Todas as questões polêmicas, de grande importância para a sociedade contemporânea, são afetadas diretamente pela religião e constantemente associadas ao conceito de moralidade (Bloom, 2012). 
Historicamente, a Psicologia da Moral aborda o tema centrando-se em duas dimensões: Justiça e Dano. No entanto, trabalhos recentes (Haidt \& Kesebir, 2010; Haidt, 2012; Haidt, 2009; Graham et al., 2011) propõem a Teoria dos Fundamentos Morais (TFM; Haidt \& Graham, 2007) que amplia o conceito de moralidade a cinco fundamentos, agregando aos dois tradicionais as dimensões: Lealdade, Autoridade e Pureza. A partir de uma revisão da literatura sobre moralidade do ponto de vista da Antropologia e da Psicologia Evolucionista (Haidt, 2009), foi proposta a TFM. De acordo com a teoria, julgamentos de certo e errado são restritos a conteúdos pertencentes aos cinco fundamentos enquanto que a predominância de um fundamento sobre outros varia entre grupos sociais (Graham et al., 2011).

A teoria dos Fundamentos Morais tem recebido bastante atenção por revelar diferenças intergrupais no julgamento moral (Haidt \& Graham, 2007). O instrumento mais utilizado para a mensuração da predominância dos fundamentos entre grupos tem sido o Questionário de Fundamentos Morais (QFM; Graham et al., 2011), porém ainda não existem evidências de validade desse instrumento para o contexto brasileiro. Nesse sentido, o objetivo deste trabalho é avaliar a validade de construto de uma versão do QMF traduzida e adaptada para o português.

A TFM articula quatro preceitos básicos (Graham et al., 2011): o nativismo, a aprendizagem cultural, a primazia da intuição e a pluralidade. A primeira afirmação diz respeito à característica inata dos fundamentos. Os fundamentos morais teriam sido naturalmente selecionados para resolver problemas sociais recorrentes no ambiente de adaptação evolutiva. A sua manifestação transcultural e nos primeiros estágios do desenvolvimento são evidências para o nativismo dos fundamentos (Haidt \& Joseph, 2007). No entanto, ainda que os fundamentos sejam inatos, eles são vulneráveis à influência do aprendizado cultural.

A segunda afirmação é que existe uma predominância de um fundamento sobre outros e que as normas sociais específicas derivadas dos fundamentos sejam definidas pela aprendizagem social. Graham et al. (2009) se referem aos fundamentos como um primeiro rascunho sobre o qual o ambiente social fará as modificações cabíveis.

A terceira afirmação é que os julgamentos morais são inicialmente guiados por processos inconscientes e automáticos. Isso se dá pela existência de dois sistemas diferentes de processamento da informação: um automático e outro controlado. O julgamento moral compreende um julgamento inicial como uma reação de gostar ou não gostar - ou de julgar positiva ou negativamente um fato (moral heuristic) - e, em seguida, uma justificativa racionalizada da reação inicial (systematic processing; Haidt, 2001).

Finalmente, os diversos problemas sociais enfrentados no ambiente de adaptação evolutiva selecionaram uma pluralidade de mecanismos cognitivos para a resolução desses problemas. Graham et al. (2012) descrevem cinco fundamentos morais que tem como função resolver problemas sociais em cinco domínios. Os cinco fundamentos são: (a) Dano (Harm) - refere-se ao sofrimento e inclui a noção de cuidado com o próximo e compaixão; (b) Justiça (Fairness) - diz respeito à noção global de justiça e direito, bem como à cooperação, competição e trapaça; (c) Pertencimento (Ingroup) - relacionado ao comprometimento com o grupo, ao autossacrifício, lealdade e vigilância contra traição; (d) Autoridade (Authority) - refere-se às obrigações relacionadas à hierarquia, obediência, respeito e cumprimento dos deveres; e (e) Pureza (Purity) - diz respeito à influência física e espiritual sobre a castidade, a salubridade e o controle dos desejos.

Os fundamentos morais são, nessa esteira, mecanismos psicológicos inatos que direcionam a aprendizagem de normas sociais. Para os autores originais, é esperado que haja outros fundamentos além dos cinco propostos (Graham et al., 2011), no entanto, os cinco fundamentos oferecem uma explicação mais parcimoniosa para as diferentes manifestações culturais de moralidade.

A teoria dos fundamentos morais tem servido como heurística para avanços teóricos e metodológicos em diversas áreas da Psicologia, incluindo a Psicopatologia, a Psicologia Política (Graham et al., 2009), e a Psicologia da Religião (Graham \& Haidt, 2010). Graham et al. (2011) encontraram que o discurso de políticos conservadores continha mais palavras correspondentes aos fundamentos de Pertencimento, Autoridade e Pureza do que o discurso de políticos liberais. Porém, Graham (2010) encontrou que pessoas com a orientação política liberal adotavam mais fortemente os mesmos três fundamentos anteriores quando eram usadas medidas de tempo de reação a palavras referentes aos fundamentos, que por autorrelato. Em um estudo que precedeu a teoria dos fundamentos morais, Haidt, Koller e Dias (1993) investigaram diferenças transculturais entre o Brasil e os Estados Unidos quanto ao julgamento moral de transgressões não danosas (que se referiam a outros fundamentos) e encontraram 
diferenças tanto entre os países em seus respectivos níveis socioeconômicos. Esses achados ilustram que os fundamentos morais influenciam o comportamento de forma antes não esperada, e que a teoria tem valor pragmático para a descoberta de novas relações na Psicologia da Moralidade.

O QMF foi desenvolvido por Graham et al. (2011) que, com uma amostra on-line de 34.476 adultos, encontrou evidências de validade interna e externa. Em uma análise fatorial exploratória com rotação direta oblíqua e estimação de máxima verossimilhança, Graham et al. (2011) encontraram seis fatores com autovalores maiores que 1. No entanto, o screeplot e as cargas dos itens indicavam que a retenção de dois fatores (um individualizante e outro coletivista) era mais interpretável.

A análise das cargas fatoriais demonstra que um item foi retido com carga abaixo de 0,3 , relacionado à justiça. A maioria (57\%) das cargas está entre 0,5 e 0,7, sendo esta a maior encontrada, e sete dos itens retidos possuem carga $\leq 0,4$. No fator 1 , as maiores cargas fatoriais foram relacionadas à decência $(0,70)$ e castidade $(0,67)$, e as menores, à família $(0,34)$ e traição $(0,48)$. No fator 2 , as maiores cargas foram para o item relacionado à fraqueza $(0,65)$ e compaixão $(0,63)$, e menores para matar $(0,35)$ e direitos $(0,47)$. Não obstante, foi possível manter os cinco fatores o que foi corroborado por análises confirmatórias.

Para avaliar se os dois fatores se decompunham nos cinco fundamentos, os autores realizaram análises fatoriais confirmatórias. Eles encontraram que o modelo contendo cinco fatores $\left(\chi^{2}=53894,1(395), \varepsilon_{\mathrm{a}}=0,046\right)$ correspondendo aos fundamentos explicava melhor os dados do que um modelo com um fator $\left(\chi^{2}=138995,4\right.$ (405), $\left.\varepsilon_{\mathrm{a}}=0,073\right)$, com dois fatores $\left(\chi^{2}=74542,9\right.$ (404), $\left.\varepsilon_{\mathrm{a}}=0,054\right)$, um Individualizante e outro Coesivo, com três fatores $\left(\chi^{2}=63074\right.$ (402), $\left.\varepsilon_{\mathrm{a}}=0,05\right)$, ou um modelo hierárquico com os fatores Individualizante e Coesivo se decompondo nos cinco fundamentos. Os cinco fundamentos tiveram consistências internas - medida pelo alfa de Cronbach e por teste-reteste - satisfatórias.

Durante o processo de investigação da validade do QMF, Graham et al. (2011) correlacionaram um indicador de comparecimento a eventos religiosos e encontraram uma relação da medida com os fundamentos Coesivos. Porém, a relação da religiosidade com os fundamentos morais seria mais bem aferida por meio de um instrumento que englobe mais dimensões da religiosidade que apenas comparecimento a cerimônias e rituais. O Índice de Religiosidade de Duke (Taunay et. al, 2012), escala adaptada para o contexto brasileiro, afere o compromisso organizacional (item 1) que trata da frequência a encontros religiosos, não organizacional (item 2) que aborda as atividades religiosas privadas e intrínseco (itens 3 a 5), que se referem à busca de vivência plena da religiosidade (Taunay et al., 2012). A utilização de um instrumento assim se justifica na medida em que oferece recursos para a discussão conceitual e por ser uma ferramenta para validação convergente para medidas implícitas. Como forma de adaptar o QMF para a pesquisa brasileira, o objetivo do presente trabalho é apresentar a adaptação do QMF para o Brasil, de forma a paramentar os pesquisadores brasileiros com uma medida confiável para o estudo dos fundamentos morais.

\section{Método}

Dado que o país não possui uma resolução específica que trate de apreciação ética de pesquisa com humanos para a área de ciências humanas e devido à natureza da presente investigação, o presente projeto não foi apreciado por um Comitê de Ética em Pesquisa. Debate sobre essa condição é conhecido na comunidade científica brasileira por meio da manifestação e ações do Fórum de Entidades de Ciências Humanas, Sociais e Sociais Aplicada (Fórum CHSSA), tendo no corrente ano, inclusive, a Sociedade Brasileira para o Progresso da Ciência (SBPC) e a Academia Brasileira de Ciências (ABC) manifestado sua favorabilidade a uma mudança substancial na maneira como a apreciação ética das pesquisas com humanos tem sido feitas no âmbito do atual único sistema existente, CEP-CONEP.

Não obstante, o presente projeto seguiu rigorosa adequação às guias éticas de pesquisa na área, como aqueles definidos pela American Psychological Association.

\section{Participantes}

Participaram da validação do instrumento 532 pessoas: 41 na validação semântica e 491 na estatística. A idade variou de 12 a 72 anos, com média igual a 29,4 $(D P=12,2)$ e mediana igual a 24 . A maioria $(41,4 \%)$ possui nível superior incompleto, seguido por superior completo $(18,9 \%)$ e segundo grau completo $(17,3 \%)$, denotando um elevado nível de escolaridade. Com relação ao estado civil, solteiros $(64,9 \%)$ e casados $(28,4 \%)$ são predominantes. O percentual de negros $(12 \%)$, pardos $(39,2 \%)$ e brancos $(45,4 \%)$ denota um equilíbrio se considerado o somatório dos dois primeiros grupos.

Dos respondentes, $80,5 \%$ declararam crer em Deus e $18,9 \%$, que não acreditam. Dado que se pretende 
aplicar o instrumento futuramente para comparar a tendência na percepção sobre moralidade entre grupos de ateístas e cristãos, ponderou-se relevante que a amostra também fosse equivalente em relação à prevalência desses grupos na sociedade.

\section{Instrumentos}

O instrumento adaptado é o Questionário de Fundamentos Morais (QMF-30), que é composto de 30 itens divididos em duas partes. Na primeira parte, a pessoa avalia cada item em termos de relevância para afirmar que algo é certo ou errado. A escala varia de 0 (zero $=$ não é nada relevante) até 5 (cinco $=$ extremamente relevante). $\mathrm{O}$ valor zero é ancorado com a frase "Esta consideração não tem nada a ver com meu julgamento de certo e errado". O valor cinco é ancorado com a frase "Este é um dos fatores mais importantes quanto eu julgo o que é certo e errado". Na segunda parte, são apresentadas 16 assertivas sobre as quais a pessoa julga o quanto concorda com cada uma delas. A escala varia de 0 (discordo fortemente) até 5 (concordo fortemente).

Os itens foram criados de modo que a pessoa manifeste o que ela considera quando rotula algo moralmente errado, sem uma referência direta de qual situação; e outra para que julgue a partir de itens contextualizados (Graham et al., 2011).

Também foi adotado, para medir o comprometimento religioso, Índice de Religiosidade de Duke que mede, além de comparecimento a cerimônias, aspectos da crença religiosa e frequência de engajamento em rituais solitários. Taunay et al. (2012) sugerem que os escores relacionados ao comprometimento intrínseco sejam computados separadamente. Assim, eles realizaram o estudo em duas amostras e, em ambas, os coeficientes alfa de Cronbach revelaram que o instrumento apresenta consistência interna adequada (amostra $1 \alpha=0,89$; IC $=95 \% ; 0,87-0,91$ e amostra $2 \alpha$ $=0,87 ; \mathrm{IC}=95 \% ; 0,83-0,91)$ para o comprometimento organizacional e não organizacional. Encontraram bons resultados também para a subescala intrínseca (amostra $1 \alpha=0,87$; IC $=95 \% ; 0,85-0,89$, e a amostra $2 \alpha=0,81$; $\mathrm{IC}=95 \% ; 0,74-0,87)$.

\section{Procedimentos}

O primeiro passo para a validação do instrumento foi realizar a tradução para o português brasileiro e a retradução para o inglês, individualmente por pessoas que dominam fluentemente ambos os idiomas - quem participou da primeira parte, não o fez na segunda.

De posse das versões, a equipe do projeto adotou o protocolo de adaptação proposto por Humbleton e
Zenisky (2011) para verificar diferentes aspectos, como questões gerais relacionadas à similaridade do significado dos termos nas duas línguas, formato do item considerando inclusive o leiaute físico, sentido geral das passagens de texto e, finalmente, elementos culturais característicos terem o mesmo grau de abstração ou familiaridade nas duas línguas.

Após cada membro do grupo preencher o instrumento, os itens foram novamente revisados em busca de problemas de compreensão. Em seguida, foi realizada uma validação semântica com 41 estudantes universitários a fim de verificar se havia itens ininteligíveis. Em inglês, os itens possuíam uma estrutura de afirmação e consequente negação, dificultando o julgamento em português, por exemplo, se alguém foi "ou não" cruel. A expressão "ou não" foi suprimida dos itens sem alterar o significado da frase.

Antes de responder ao questionário, foi obtido junto aos participantes um assentimento, em que as pessoas foram informadas sobre propósitos e outros elementos da pesquisa, mas não foi solicitado do participante a assinatura de qualquer tipo de consentimento. A opção pelo assentimento deve-se ao fato de que, dessa forma, os objetivos com relação ao esclarecimento sobre a pesquisa foram atingidos $\mathrm{e}$ os aspectos ético/morais preservados sem fornecer detalhes sem fornecer a priori elementos que possam influenciar o julgamento com relação aos itens do instrumento ora validado.

De posse da versão final, outros participantes responderam ao QMF tanto em suporte papel quanto em formato eletrônico. $\mathrm{Na}$ aplicação presencial, as pessoas foram abordadas em seu local de estudo ou de trabalho e solicitadas a responder ao questionário de comprometimento religioso (DUKE), às questões demográficas e finalmente ao QMF. A versão virtual foi construída no EFS Survey (http://www.unipark.com) e o link encaminhado a estudantes de duas instituições de ensino superior do Distrito Federal, obedecendo à mesma estrutura da aplicação presencial.

Foram realizadas análises preliminares para testar a adequabilidade da amostra e a análise paralela a fim de decidir o número de fatores a serem extraídos no IBM SPSS Statistics. Com base nesses resultados, foram testados via análise fatorial exploratória modelos com 3, 5 e 6 fatores. Finalmente, com base nos procedimentos de Graham et al. (2011) foram extraídos dois fatores. Como forma de aferir a validade convergente foi realizada uma correlação bivariada entre os escores fatoriais da solução fatorial da versão brasileira do QMF e os itens da DUKE.

Psico-USF, Bragança Paulista, v. 21, n. 3, p. 487-495, set./ dez. 2016 


\section{Resultados}

As análises preliminares apontam para a adequabilidade da amostra, dado que o KMO $(0,928)$ pode ser considerado excelente (Field, 2013). Todas as variáveis possuem medida de adequação da amostra (Measure of Sampling Adequacy - MSA) $>0,05$, indicando ajuste à estrutura das demais variáveis e ausência de multicolinearidade. O tamanho da amostra e a média da comunalidade $(0,59)$ permitem aceitar o critério de Kaiser como preciso.

Assumindo o valor próprio maior que um e sem rotação, sugeriu seis dimensões que explicam 59\% da variância total. O screeplot e a análise paralela, cuja adoção é fortemente sugerida por Laros (2005), apontaram para uma estrutura com três fatores.

Com base nesses resultados, optou-se a partir do critério de Kaiser (manter fatores com autovalor maior que 1) investigar a melhor adequação da fatoração dos eixos principais extraindo três e seis fatores, com rotação oblíqua assumindo a relação entre as dimensões como proposto por Graham et al. (2011). Além disso, em função da estrutura teórica preconizada para o QMF, foi testada a estrutura com cinco fatores correspondendo aos cinco fundamentos morais. A Fatoração dos Eixos Principais (PAF) com seis fatores possui variância acumulada 48,59\%, com apenas dois fatores apresentando valor próprio 1 . A matriz-padrão mostra a necessidade de retirar quatro itens e dois fatores que apresentam apenas um item. O fator 1 e o fator 6 apresentaram o maior número de itens retidos $(10$ e seis respectivamente).

A PAF com cinco fatores, suportada teoricamente pela Teoria dos Fundamentos Morais, possui explicação de variância de $46,59 \%$ e apenas dois fatores tiveram valor próprio $>1$. A análise da matriz-padrão mostram dez itens a serem suprimidos e dez agrupados no fator 1. Os demais fatores ficaram com quatro, um e dois itens respectivamente.

A PAF com três fatores, sustentada pela interpretação do screeplot e pela Análise Paralela, apresentou uma explicação da variância acumulada de 42,01\%, sendo que dois dos fatores apresentaram valor próprio $>1$. A análise da matriz-padrão mostra quatro com sugestão de retirada. Os fatores 1 e 2 apresentaram retenção de 12 e 13 itens e o terceiro fator apenas um.

Graham et al. (2011) verificaram e testaram uma tendência de agrupamento dos itens em dois fatores: um chamado Individualizante que agrega os itens elaborados para dano e justiça (DJ) e outro Coesivo, que agrega pertencimento, autoridade e pureza (PAP). Nessa esteira, embora a TFM sugira cinco fundamentos, é coerente também testar essa estrutura. Assim, foi rodada uma PAF com extração de dois fatores e rotação Oblimin, com supressão de cargas fatoriais $<0,30$.

Dos 30 itens iniciais, foram retidos 27 (12 para o primeiro fator e 15 para o segundo). Dos itens retirados, dois eram do fundamento Autoridade: "Se alguém mostrou falta de respeito por autoridade" e "Se alguma ação causou caos ou desordem" e um de Justiça: "Eu acho que é moralmente errado crianças ricas herdarem muito dinheiro enquanto crianças pobres não herdam nada". Dois itens de Pertencimento no questionário original ("Se alguém mostrou falta de lealdade" e "Se alguém fez alguma coisa para trair o seu grupo") se enquadraram no conjunto DJ.

Não obstante, nem a retirada dos três itens e nem a presença de assertivas do grupo contrário modificaram a estrutura lógica dos construtos em dois fatores já que o fator Individualizante ainda é composto $83,3 \%$ de Dano e Justiça e o Fator coletivista contém 93,3\% de itens construídos para Pertencimento, Autoridade e Pureza.

Para testar a relação entre o QMF e o Duke, foi criado um índice da diferença entre os fatores (F1-F2). Esse índice representa a tendência Individualizante ou Coesiva, sendo que quanto maior o valor encontrado maior a tendência de prevalência do fator Individualizante. Dado que o Duke afere a religiosidade (Taunay et al., 2012) e pressupõe-se que o fator Individualizante prepondera no julgamento de ateístas, espera-se que nessa amostra a diferença F1-F2 não apresente correlação com os fatores do Duke enquanto o oposto ocorra na amostra teísta. A Tabela 2 mostra essas correlações.

Ao encontro com o esperado, a maior correlação encontrada foi entre F1-F2 com religiosidade intrínseca na amostra de teístas $(r=0,43, p<0,01)$. Também foi identificada correlação moderada e significativa $(r$ $=0,36, p<0,01) \mathrm{com}$ religiosidade organizacional. As correlações na amostra ateísta foram todas negativas sendo duas moderadas e não significativas com religiosidade não organizacional $(r=-0,33)$ e intrínseca $(r=-0,32)$.

\section{Discussão}

O objetivo do trabalho foi adaptar o QMF para o português de tal forma que, ao final, tenha-se um instrumento que discrimine a tendência de julgamento de 
Tabela 1

Cargas Fatoriais da Análise Fatorial Exploratória Por Itens

\begin{tabular}{|c|c|c|}
\hline & \multicolumn{2}{|c|}{ Fatores } \\
\hline & $\mathrm{HF}$ & IAP \\
\hline F - Se alguém teve seus direitos negados. & 0,81 & \\
\hline F - Se alguém agiu de forma injusta. & 0,74 & \\
\hline H - Se alguém foi não cruel. & 0,74 & \\
\hline F - Se algumas pessoas foram tratadas diferente das outras. & 0,67 & \\
\hline H - Se alguém sofreu emocionalmente. & 0,66 & \\
\hline I - Se alguém mostrou falta de lealdade. & 0,64 & \\
\hline H - Se alguém cuidou ou não de alguém fraco ou vulnerável. & 0,63 & \\
\hline I - Se alguém fez alguma coisa para trair o seu grupo. & 0,59 & \\
\hline F - A justiça é o requisito mais importante para uma sociedade. & 0,55 & \\
\hline $\begin{array}{l}\text { F - Quando o governo cria leis, o princípio fundamental deve ser garantir que todos sejam } \\
\text { tratados de forma justa. }\end{array}$ & 0,52 & \\
\hline H - Compaixão por aqueles que estão sofrendo é a virtude mais importante. & 0,50 & \\
\hline H - Uma das piores coisas que alguém poderia fazer é ferir um animal indefeso. & 0,38 & \\
\hline P - Se alguém agiu ou não de um jeito que Deus aprovaria. & & 0,72 \\
\hline P - As pessoas não deveriam fazer coisas nojentas, mesmo que ninguém seja prejudicado. & & 0,69 \\
\hline P - A castidade é uma virtude importante e valiosa. & & 0,62 \\
\hline $\begin{array}{l}\text { A - Se eu fosse um soldado e discordasse do meu comandante, eu o obedeceria de } \\
\text { qualquer forma, pois esse é o meu dever. }\end{array}$ & & 0,59 \\
\hline P - Eu julgaria alguns atos como errados, alegando que não são naturais. & & 0,58 \\
\hline P - Se alguém violou ou não padrões de pureza e decência. & & 0,55 \\
\hline I - As pessoas deveriam ser leais a seus familiares, mesmo que tenham feito algo errado. & & 0,55 \\
\hline A - Homens e mulheres têm papéis diferentes para desempenhar na sociedade. & & 0,52 \\
\hline I - Se as ações de alguém mostraram ou não amor por seu país. & & 0,50 \\
\hline A - Se alguém se adequou ou não às tradições da sociedade. & & 0,49 \\
\hline H - Nunca pode ser correto matar um ser humano. & & 0,46 \\
\hline P - Se alguém fez ou não algo nojento. & & 0,41 \\
\hline I - Eu tenho orgulho da história do meu país. & & 0,40 \\
\hline I - É mais importante trabalhar em equipe do que agir individualmente. & & 0,39 \\
\hline A - Respeito por autoridade é algo que todas as crianças precisam aprender. & & 0,39 \\
\hline & $\alpha=0,91$ & $\alpha=0,87$ \\
\hline & $\sigma^{2}=26,01$ & $\sigma^{2}=8,95$ \\
\hline
\end{tabular}

acordo com a Teoria dos Fundamentos Morais. Pressupondo que a moralidade se divide nos cinco domínios propostos e que estes se relacionem de forma diferenciada com uma medida de religiosidade, espera-se que, numa amostra brasileira, o QMF meça cinco variáveis latentes correspondentes aos fundamentos morais $\mathrm{e}$ que os fundamentos de coesão social tenham uma correlação positiva com a medida de religiosidade.

Os resultados encontrados vão ao encontro dos obtidos por Graham et al. (2011) onde o instrumento se dividiu em dois fatores (Individualizante - HF e Coesivo - IAP). A diferença centrou-se em dois itens de 
Tabela 2

Correlação QMF - DUKE

\begin{tabular}{lcccccccccc}
\hline & & \multicolumn{3}{c}{ Geral } & \multicolumn{4}{c}{ Acredita em Deus } & \multicolumn{2}{c}{ Não acredita em Deus } \\
\hline & F1-F2 & RO & RN & RI & RO & RN & RI & RO & RN & RI \\
F1-F2 & 1 & $0,312^{*}$ & 0,176 & $0,416^{*}$ & $0,361^{*}$ & 0,157 & $0,433^{*}$ & $-0,157$ & $-0,33$ & $-0,315$ \\
RO & $0,312^{*}$ & 1 & $0,517^{*}$ & $0,607^{*}$ & 1 & $0,375^{*}$ & $0,462^{*}$ & 1 & 0,195 & 0,426 \\
RN & 0,176 & $0,517^{*}$ & 1 & $0,629^{*}$ & $0,375^{*}$ & 1 & $0,446^{*}$ & 0,195 & 1 & 0,171 \\
RI & $0,416^{*}$ & $0,607^{*}$ & $0,629^{*}$ & 1 & 0,462 & $0,446^{*}$ & 1 & 0,426 & 0,171 & 1 \\
\hline
\end{tabular}

$* p<0,01$

F1-F2 - Índice da diferença entre os fatores.

$\mathrm{RO}$ - Religiosidade organizacional.

$\mathrm{RN}$ - Religiosidade não organizacional.

RI - Religiosidade intrínseca.

pertencimento que foram retidos no fator Individualizante e um de dano no Coesivo. Nesses casos, é factível crer que as palavras "lealdade" e "traição" ativem um referencial semântico mais fortemente associado a (in) justiça do que para pertencimento nesta amostra. O mesmo ocorre com um item de dano ("Nunca pode ser correto matar um ser humano") que ficou no grupo IAP. A frase como está redigida pode ser associada a um mandamento religioso, podendo ser interpretado no contexto como próximo à pureza e à autoridade. Uma explicação alternativa é que o deslocamento dos itens seja fruto de diferenças culturais entre as amostras, tendo em vista as origens diferentes dos participantes do estudo de Graham et al. (2011) (majoritariamente estadunidense) e a brasileira.

Embora reduzido a dois grandes fatores, os resultados permitem discriminar tendências distintas com relação à atitude das pessoas diante de uma situação que elicia um julgamento moral. Aplicado em concomitância com uma escala de comprometimento religioso, percebeu-se, ao contrastar teístas e ateístas, que o fator Individualizante está negativamente correlacionado aos não crentes.

Longe da polêmica de que a religião é o fundamento da moralidade ou se ela e a crença em Deus se torna de fato obstáculos ao comportamento ético (Haidt \& Kesebir, 2010), não é absurdo supor que exista alguma diferença na forma pela qual teístas e ateístas julgam se um ato é ou não moral. Não por acaso Shweder propõe três alegações do desenvolvimento moral (Bruce, 2013) e há uma específica para divindade. A TFM, apesar de incorporar a lógica de ter um fundamento (pureza/santidade) na qual se espera maior ligação com a religiosidade, amplia essa relação ao assumir seu caráter de integração e coesão (Haidt \& Kesebir, 2010).

É dessa forma que Graham et al. (2011) entendem a junção dos fundamentos de autoridade, pertencimento e pureza em um único fator Coesivo. Essa perspectiva se alinha ao entendimento da religião como passível de uma definição extrapolando a crença em sobrenatural e/ou no pressuposto de uma divindade indo ao encontro da religião como uma atividade social, de pertencimento (Bloom, 2012). O fato da versão adaptada ao português reter os dois fatores e encontrar relação com o comprometimento religioso é, então, relevante.

Este trabalho permite concluir que a versão adaptada, aqui apresentada, tem indícios de validade, o que possibilitam o seu uso em atividades de pesquisa, em amostras brasileiras, que tenham como interesse investigar os fundamentos morais. Fica evidente que o trabalho de desenvolvimento dessa versão adaptada ainda merece atenção dos pesquisadores interessados, mas considera-se que essa primeira versão tem potencial para o incremento dos estudos sobre fundamentos morais em amostras brasileiras, atividade ainda incipiente nos estudos da Psicologia Moral no país.

\section{Referências}

Bloom, P. (2011). Family, community, trolley problems, and the crisis in moral psychology. The Yale Review, 99, 26-43. doi: 10.1111/j.1467-9736.2011.00701.x

Bloom, P. (2012). Religion, Morality, Evolution. Annu. Rev. Psychol, 63,179-99. doi: 10.1146/ annurev-psych-120710-100334 
Bruce, J. R. (2013). Uniting theories of morality, religion, and social interaction: grid-group cultural theory, the "Big Three" ethics, and moral foundations Theory. Psychology \& Society, 5, 37-50. Recuperado de http: / www.psychologyandsociety. org/__assets/_original/2013/02/Bruce_J.pdf

Field, A. P. (2013). Discovering statistics using IBM SPSS Statistics: And sex and drugs and rock ' $n$ ' roll (fourth edition). London: Sage publications.

Graham, J., Nosek, B. A., Haidt, J., Iver, R., Koleva, S., \& Ditto, P. H. (2011). Mapping the moral domain. Journal of Personality and Social Psychology, 101, 2, 366385. doi: $10.1037 / \mathrm{a} 0021847$

Graham, J., Haidt, J., \& Nosek, B. A. (2009). Liberals and conservatives rely on different sets of moral foundations. Journal of Personality and Social Psychology, 96, 5, 1029-1046. doi: 10.1037/a0015141

Graham, J., \& Haidt, J. (2010). Beyond beliefs: Religion binds individuals into moral communities. Personality and Social Psychology Review, 14, 140-150. doi: 10.1177/1088868309353415

Haidt, J., Koller, S., \& Dias, M. (1993). Affect, culture, and morality, or is it wrong to eat your dog? Journal of Personality and Social Psychology, 65, 613-628. doi: 10.1037/0022-3514.65.4.613

Haidt, J. (2001). The emotional dog and its rational tail: A social intuitionist approach to moral judgment. Psychological Review, 108, 814-834. Recuperado de http://www.motherjones.com/files/emotional_ dog_and_rational_tail.pdf

Haidt, J., \& Joseph, C. (2007). The moral mind: How 5 sets of innate moral intuitions guide the development of many culture-specific virtues, and perhaps even modules. Em P. Carruthers, S. Laurence, and
S. Stich (Eds.) The Innate Mind. New York: Oxford, 367-391.

Haidt, J., \& Graham, J. (2007). When morality opposes justice: Conservatives have moral intuitions that liberals may not recognize. Social Justice Research, 20, 98-116. doi: 10.1007/s11211-007-0034-z

Haidt, J. (2009). Moral psychology and the misunderstanding of religion. Em J. Schlosse M. Murray (Eds.), (2009). The believing primate: Scientific, philosophical, and theological reflections on the origin of religion. New York: Oxford, 278-291.

Haidt, J., \& Kesebir, S. (2010). Morality. Em Handbook of Social Psychology, ed. S. Fiske, D. Gilbert, 797-832. New York: MacGraw Hill. 5th ed.

Haidt, J. (2012). The Righteous Mind: why Good People Are Divided by Politics and Religion. New York: Pantheon.

Humbleton, \& Zenisky (2011).Translating and Adapting Tests for Cross-Cultural

Assessments. In D. Matsumoto, F. J. R. van de Vijver (Eds.)Cross-cultural research methods in psychology. New York: Cambrige University Press, 46-70.

Laros, J. A. (2005). O uso de análise fatorial: Algumas diretrizes para pesquisadores. Em L. Pasquali (Eds.), Análise fatorial para pesquisadores (pp. 163-184). Brasília: LabPAM

Taunay et al. (2012). Validação da versão brasileira da escala de religiosidade de Duke (DUREL). Revista de Psiquiatria Clínica, 39(4), 130-135. Recuperado de http://www.scielo.br/pdf/rpc/v39n4/03.pdf

Recebido 20/04/2015

Reformulado 11/12/2015

Aceito 11/01/2016 
Sobre os autores:

Alexandre Magno Dias Silvino é doutor em Psicologia pela Universidade de Brasília (UnB), pós-doutorando no Programa de Psicologia Social, do Trabalho e das Organizações (PSTO) da UnB e pesquisador tecnologista do Instituto Nacional de Estudos e Pesquisas Anísio Teixeira.

E-mail: alexandre.silvino@gmail.com

Ronaldo Pilati possui doutorado em Psicologia pela Universidade de Brasília e é professor adjunto do Departamento de Psicologia Social e do Trabalho (PST) e do Programa de Pós-Graduação em Psicologia Social, do Trabalho e das Organizações (PSTO) da UnB.

E-mail:rpilati@gmail.com

Victor Hahuel Keller é graduando em Psicologia pela Universidade de Brasília e bolsista de Iniciação Científica do PROIC/CNPq.

Elena Pinheiro Silva é graduanda em Psicologia pela Universidade de Brasília, bolsista do Programa Jovens Talentos para a Ciência pela CAPES e pesquisadora do Grupo de Estudos em Psicologia Social da UnB.

Aline Fernandes de Paula Freitas é graduanda em Psicologia pela Universidade de Brasília e bolsista de Iniciação Científica do PROIC/CNPq.

Juliana Nunes Silva é graduanda em Psicologia pela Universidade de Brasília e pesquisadora do Grupo de Estudos em Psicologia Social da UnB.

Maíra Fernandes Lima é graduanda em Psicologia pela Universidade de Brasília e bolsista do Programa Jovens Talentos para a Ciência pela CAPES.

Contato com os autores:

Alexandre Magno Dias Silvino, INSTITUTO NACIONAL DE ESTUDOS E PESQUISAS EDUCACIONAIS ANÍSIO TEIXEIRA - INEP, SIG Quadra 04 - Lote 327, Edifício Villa Lobos, Brasília, Brasil

CEP: 70610-440

Fone/Fax: (61) 2022-3443/ (61) 9203-0116

E-mail: alexandre.silvino@gmail.com 


\section{ERRATA}

No artigo Adaptação do Questionário dos Fundamentos Morais para o Português, com número de DOI: $<10.1590 / 1413-82712016210304>$, publicado no periódico Psico-USF, 21(3): páginas 487-495, na página 495 faltou a seguinte informação:

O presente trabalho recebeu apoio do CNPq na forma de Bolsa Produtividade em Pesquisa (PQ) e apoio individual no Edital Universal CNPq (478285/2013-2) para o segundo autor. 